\title{
No advantage for separating overt and covert attention in visual search
}

W. Joseph MacInnes ${ }^{1,4}$, Ómar I. Jóhannesson ${ }^{2}$, Andrey Chetverikov ${ }^{3}$, \& Árni Kristjánsson ${ }^{1,2}$

1) School of Psychology, National Research University Higher School of Economics, Russia

2) Icelandic Vision Laboratory, Department of Psychology, University of Iceland

3) Donders Institute for Brain, Cognition, and Behaviour, Radboud University, Nijmegen, Netherlands

4) Vision Modelling Lab, Faculty of Social Sciences, National Research University Higher School of Economics, Moscow, Russia

Corresponding Author:

W. Joseph MacInnes

Jmaclnnes@hse.ru

OrcID: 0000-0002-5134-1601

Funding provided in part, by

-Research Laboratory Grant, Vision Modelling Lab, provided by the National Research University Higher School of Economics 


\section{Abstract}

We move our eyes roughly three times every second while searching complex scenes, but covert attention helps to guide where we allocate those overt fixations. Covert attention may be allocated reflexively or voluntarily, and speeds the rate of information processing at the attended location. Reducing access to covert attention hinders performance, but it is not known to what degree the locus of covert attention is tied to the current gaze position. We compared visual search performance in a traditional gaze contingent display with a second task where a similarily sized contingent window is controlled with a mouse allowing a covert aperture to be controlled independently from overt gaze. Larger apertures improved performance for both mouse and gaze contingent trials suggesting that covert attention was beneficial regardless of control type. We also found evidence that participants used the mouse controlled aperture independently of gaze position, suggesting that participants attempted to untether their covert and overt attention when possible. This untethering manipulation, however, resulted in an overall cost to search performance, a result at odds with previous results in a change blindness paradigm. Untethering covert and overt attention may therefore have costs or benefits depending on the task demands in each case. 


\section{Introduction}

The visual field contains, at any given moment, far more information than our visual system can process in detail. We therefore use attention to select items for further processing, that are of potential importance (Desimone \& Duncan, 1995; Egeth, 1977; see Driver, 2001 for a review). This may entail costs, however, as unattended items can often go unnoticed and we can miss surprisingly salient events in our visual field if our visual attention is not directly applied to them (Rensink et al., 1997; Most et al., 2005; Kristjánsson, 2006). At the same time other stimuli can break through the clutter and capture our attention even when this goes against our goals (Egeth \& Yantis, 1997; Theeuwes, 2010).

\section{Overt and covert attention}

The fovea centralis contains the highest density of cones on the retina and is responsible for our high resolution vision. The locus of attention often overlaps with our centre of gaze and it is natural to ensure that the location or item that we wish to scrutinize falls on the fovea. We move our eyes roughly three times a second to overtly shift our centre of gaze to the attended location. But we can also attend to locations in space without fixating them directly, and such covert orienting also produces behavioural changes at the attended location (Posner, 1980). Such covert attending was probably first systematically investigated by Hermann von Helmholtz. He used a darkened room where only a small pinhole was visible that he kept fixation on (Von Helmholtz,1867). Helmholtz discovered that he was able to select a region, outside of the center of his gaze, and pay specific attenton to that region. When the room was briefly illuminated with a flash, he was able to recognize letters in that region even though his gaze was centered elsewhere, while other letters at unattended locations were a blur. This revealed the existence of an attentional mechanism that was spatially selective but did not involve eye movements and increased visual resolution at the attended location.

Other evidence suggests that attention and eye movements are tightly coupled, so that attention can be typically expected to move to the locus of gaze (Deubel \& Schneider, 1996; Hoffmann \& Subramaniam, 1995; Kowler et al., 1995). In fact, attention has been proposed to be intrinsically coupled with the oculomotor system (Oculomotor readiness - Klein, 1980; Premotor theory - Sheliga, Riggio \& Rizzolatti, 1994; see review in Kristjánsson, 2011). However the relationship between attention and eye movements is, probably, not as all-or-none as is often implied (Klein \& Pontefract, 1994; Hunt \& Kingstone, 2003; Sato \& Schall, 2003; Wollenburg, Deubel \& Szinte, 2018; Smith \& Schenk, 2012). Although attention and eye movement networks do have significant overlap (Beauchamp et al., 2001; Corbetta et al., 1998; de Haan, Moryan, \& Rorden, 2008; Kustov \& Robinson, 1996), the differences are often as telling as the similarities. The frontal eyefields (FEF) are involved in both attention and eye movements with single-cell stimulation producing saccades with high intensity stimulation and attention benefits with low intensity stimulation (Armstrong et al, 2006). Similarly, TMS to the FEF disrupts saccadic programming (Beckers et al, 1992) and spatial attention in cueing (Grosbras \& Paus, 2002) and search (Muggleton et al, 2003). Within the

FEF, however, different populations mediate covert and overt attention (Sato \& Schall, 2003) and may control attention and saccades independently (Thomson et al, 2005). Additionally, the findings of Chica et al., (2010) suggest that endogenous saccade preparation is, on it's own, not suffiecient to observe well-known attention effects 
like inhibition of return (IOR). In sum, while attention and eye movements often work together, they reflect separate mechanisms and can therefore be uncoupled.

\section{Untethering Overt and Covert attention}

The literature has up until now not offered paradigms that allow researchers to untether covert and overt attention while still allowing observers to move their eyes. Studies of covert attending in the literature typically require fixation and a peripheral task, as in the example from Helmholtz stated above (see e.g. Posner, 1980; Kristjánsson \& Nakayama, 2003; Engbert \& Kleigl, 2003; Corrasco \& NcElree, 2001) but may also involve manipulating the amount of peripheral information available to the participant though gaze contingent displays (Foulsham \& Underwood, 2011; Geisler, Perry, \& Najemnik, 2006).

In previous work, we introduced a paradigm that allows a dissociation between covert and overt attention. We compared covert and overt contributions to a change blindness task using mouse-contingent and gaze-contingent displays (Chetverikov et al., 2018). Observers had to judge whether a change occurred somewhere in an array of objects between two alternating presentations of the array. The array was covered with a grey mask, but observers could uncover portions of the array of stimuli through a Gaussian-shaped aperture by moving their gaze or by moving a mouse. While the gaze-contingent condition tethered the (covertly) viewable area to the centre of gaze, the mouse-contingent condition allowed participants to untether the covert viewing area from their focus of gaze, while keeping overall viewable area size the same. Using this approach, Chetverikov et al. investigated the potential costs and benefits of allowing observers to untether the two forms of attention. The results were that observers searched for the changing item differently when they could deploy covert attention separately from gaze position, in a seeming effort to use covert attention. Importantly, change detection was faster when overt and covert attention were uncoupled, indicating that there were benefits to this untethering of attention and gaze.

\section{Current aims}

If voluntary attention can be applied both overtly and covertly, we can expect that performance on tasks like visual search might benefit under conditions where the two can be deployed separately, compared to when the two maintain a single focal point. For example, when items in a search array can be dismissed based on a feature distinguishable with covert attention (like colour), overt attention could be deployed to the remaining subset to improve search efficiency. While it is quite natural for our attention to follow our gaze, our aim was to investigate voluntary visual attention using a paradigm where covert and overt attention can be untethered.

Attention has been studied extensively in various visual search tasks (Duncan \& Humphreys, 1989; Kristjánsson, 2015; Nakayama \& Martini, 2011; Neisser, 1964). Here we attempt to address the question of what contribution covert attention makes to visual search and when it can be allocated independently of overt attention.

We used visual search displays where observers had to search for a target with a certain shape and color and a specific symbol within the shape. Examples of such "alien traffic sign" stimuli are shown in figure 1. Twenty-four such items appeared on the screen but were covered by a grey mask that could be revealed through an aperture controlled by 1) gaze movement or 2) the movement of a mouse cursor. That is, the visible area for all trials was restricted and contingent on either mouse position or gaze position. The latter mouse contingent condition in 
particular allowed the uncovering of items independently of eye movements while otherwise keeping the two conditions as similar as possible. We used two aperture sizes, either 5 or 9 degrees of visual angle.

In our gaze contingent paradigm, moving gaze around moved the mask. We compared such a paradigm with our newly designed mouse-contingent paradigm (Chetverikov et al., 2018) where the visual display changes according to the real-time location of a user-controlled mouse cursor, allowing comparison of visual search performance with mainly overt attention (gaze-contingent display) and untethered overt and covert attention (mouse-contingent display).

We ask the following questions: Firstly, do observers try to utilize this covert attention that is simulated with mouse movements that observers control themselves during a visual search task, or do observers volitionally "tether" gaze and mouse position? Secondly, can observers use this possibility of untethered covert and overt attention to improve their performance during a visual search task? We assessed this by looking at performance (response time and accuracy) and tracking observers' gaze as they performed the visual search task. Thirdly, what insights can our paradigm provide about the contribution of overt and covert attention to visual search performance?

\section{Methods}

Twenty participants were recruited from the University of Iceland student population and received course credit for their participation. One participant was removed from analyses because of chance level performance in some of the experiment conditions. The study was conducted in line with the requirements of the appropriate ethics committee, and all participants signed informed consent.

The movements of the right eye were tracked at $500 \mathrm{~Hz}$ with an EyeLink 1000+. The stimuli were presented on a 24 inch wide monitor (BenQ, model XL24112) with a resolution of 1920 x 1080 pixels running at $60 \mathrm{~Hz}$. The distance from the eye to the screen was $100 \mathrm{~cm}$. The computer used to control the presentation had an "Intel Core i7" $4 \mathrm{GHz}$ processor and 8 GB RAM running Windows 7 Professional 64-bit. Head movements were restricted with a head and chin rest.

The participants' task was to search for a unique target in a 4 row by 6 columns matrix of items and respond with a keyboard press whether the target was present or absent. Search items were chosen from the set used by Chetverikov (2018) (see figure 1 for examples) and could vary by shape (diamond or square), colour (blue or brown) and/or symbol (64 possible drawn targets). The target was the one containing the unique symbol (see figure 1). Each trial was initiated by the participant with a button press and drift correction while looking at a central fixation. This was immediately followed by the presentation of the search target at fixation for one second. This sample target was shown complete with shape, colour and symbol information, even though it was possible to find the target by symbol alone. Mask, aperture and trial stimuli representing the full search array were then presented in a four-row by six-column grid. The width and height of each stimulus was 2 degrees visual angle (dva) with 2 dva spacing between each row and column. Target absent trials (66.7\% of each block) had equal likelihood of each colour and shape, and the set of symbols was randomly drawn from the full set with the stipulation that symbols were not repeated regardless of colour and shape. Target present trials $(33.3 \%)$ were similar except that a random item was replaced with the actual target. Although the original intent was to present $50 \%$ target present trials, a coding error 
resulted in the biased percentages reported here. No other problems resulted from this error, and both colours and both shapes were equally likely as distractors on every trial. The non-duplication of symbol within each trial meant that participants could employ a strategy of searching a subset of the items presented based on colour and/or shape, but they could also choose to ignore this information and perform the search based on the symbol alone. A trial lasted for a maximum of 8 seconds, or until the participant responded with a key press (left/right arrow for target present/absent).

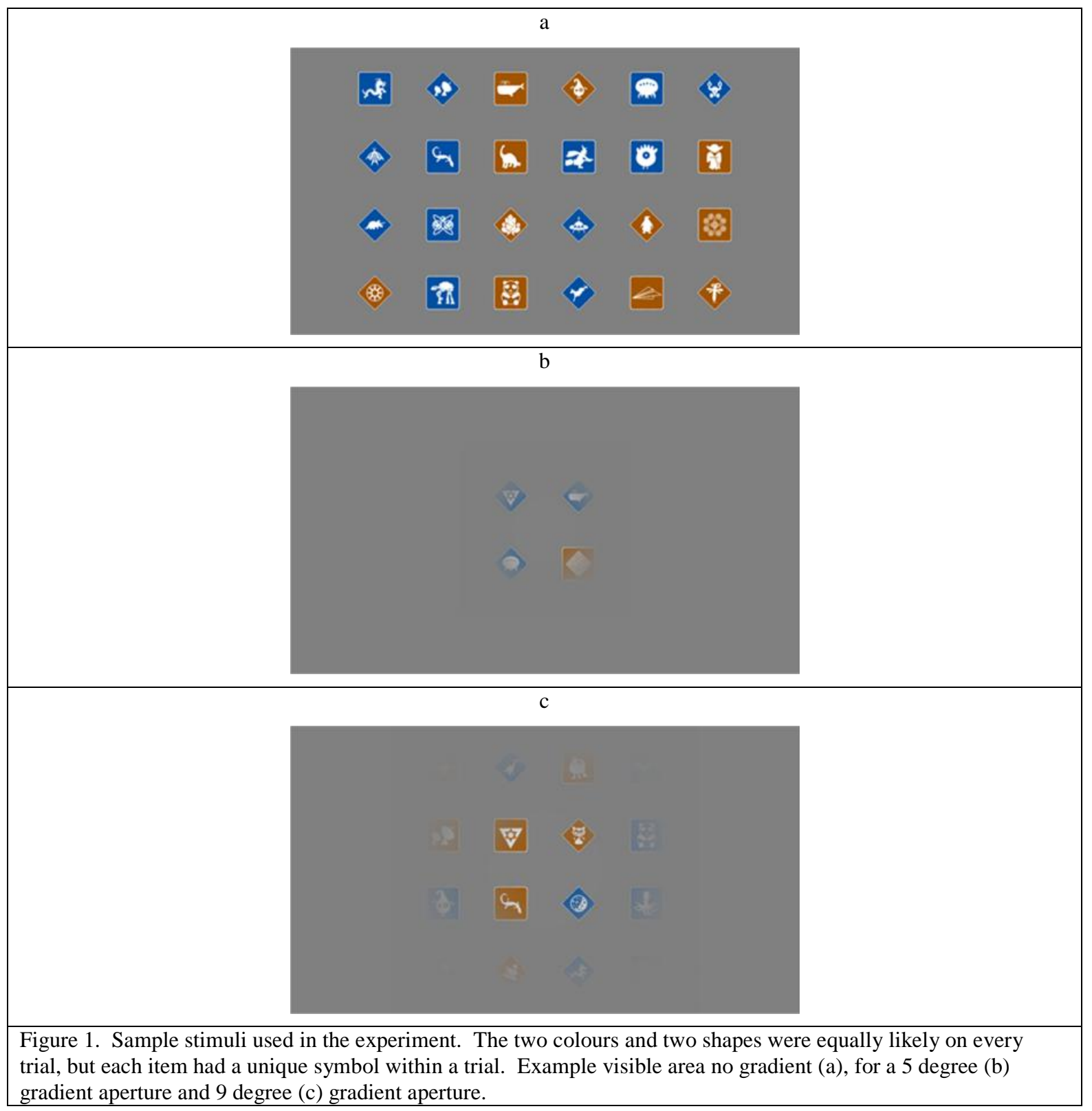

All participants contributed data to five different blocks of trials with 72 trials per block. The viewable search area differed for each block with one having the full search array visible throughout each trial and the other four having only part of the screen visible through a participant-controlled aperture. These four aperture blocks consisted of each combination of two factors: aperture size ( 5 or 9 degree diameter visible), and aperture-control (gaze or mouse). The visible area of the aperture involved a gaussian gradient (See appendix a for specific parameters) 
toward the edges so that an additional one degree was partially visible to give the aperture a softer edge (see figure 1b). Gaze aperture control fixed the centre of the aperture to the participants' gaze location, while the mouse control allowed separation of gaze and aperture by controlling its centre with the mouse. The baseline condition was run first for all participants to familiarize them with the task and search stimuli, while four aperture blocks were presented afterwards, according to a Latin square design. Each aperture block was also preceded by 10 practice trials to allow observers to adapt to the various aperture controls. Aperture location was determined by the experiment code just prior to each monitor frame refresh $(60 \mathrm{~Hz})$ and redrawn with its centre at the most recent location of the participants' gaze or mouse.

\section{Results}

Our primary performance measures of visual search performance were accuracy of response and response times for trials with correct responses. We used these measures to determine the benefit, if any, of untethered covert attention in the mouse contingent condition. Additionally, we calculated a number of eye-tracking metrics to determine whether there was any evidence that participants chose to, or were able to deploy such a strategy of untethering covert attention.

Response accuracy was analysed with a generalized linear model (binomial family) using the lme4 package (Bates et al. 2015). Statistical significance of the effects was estimated through model comparison using ANOVA $\chi 2$ tests. Subject, target colour and target shape were included as crossed random factors with target information additionally included as intercepts. We anticipated potential interactions between aperture type and aperture size so both variables were included as fixed effects in addition to whether the target was present on that trial. The accuracy at the baseline with target present and a 9 dva aperture was 86.1 (percent). Target absent trials were significantly more accurate $\left(+8.6 \%, \chi^{2}(1)=175, \mathrm{p}<.001\right)$ and also interacted $(\chi 2(4)=55, \mathrm{p}<.001)$ with the mouse 


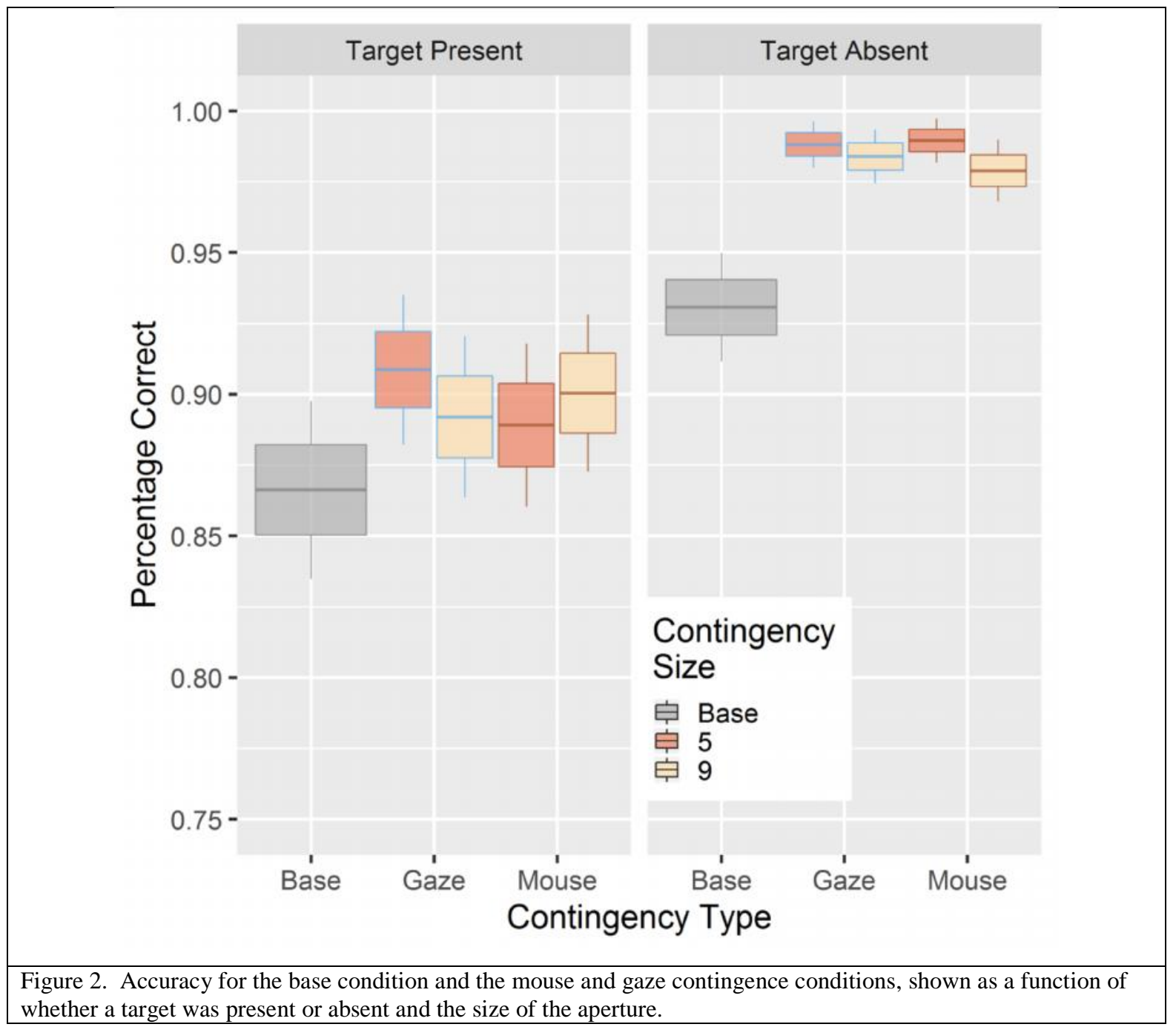

contingent condition with mouse and gaze gaining additional accuracy over baseline when the target was absent (see figure 2). No other interactions or main effects were significant with a final model of (correct Contingency:ProbeTrial+ProbeTrial+ProbeColour + ProbeShape $+(1 \mid$ ProbeShape $)+(1 \mid$ ProbeColour $)+$ $(1 \mid$ Subject $))$ in the lme4 syntax. A second lme removing the baseline condition showed only a main effect of whether a target was present or not with no significant interactions. The high accuracies in the reduced (aperture) viewing conditions suggest a speed accuracy trade-off, but this was only in contrast to the baseline condition and not within the various different apertures. Due to the high accuracies and lack of key interactions, we decided to focus primarily on response time as our primary measure of performance.

Response times (RTs) were analyzed with the same variable selection as accuracy, though with baseline trials removed and with the Gaussian lme family. Baseline trials are plotted (figure 3) but removed from analyses due to their obvious performance advantage and since they do not have different aperture sizes for exploring our key interactions. Alternate models are compared using a chi-square test to determine if additional variables significantly improve the overall model fit. The RT intercept for gaze contingent trials for the 5 dva aperture was 2.58 seconds with a main effect of target present $(\chi 2(1)=1580, p<.001)$ with responses on target absent trials being 1.54 seconds slower than target present trials $(S E=0.03 \mathrm{~s})$. The key main effects were significant; contingency type $(\chi 2(1)=148, p$ 
$<.001)$ with slower responses in the mouse contingent than gaze-contingent condition $(+0.42 \mathrm{~s}, \mathrm{SE}=0.03)$, and faster responses $\left(\chi^{2}(1)=8.4, \mathrm{p}=.004\right)$ for the $9 \mathrm{dva}$ aperture than the $5 \mathrm{dva}(-0.10 \mathrm{~s}, \mathrm{SE}=0.03)$. None of the interactions were significant with the final model $(R T \sim$ ProbeTrial + Contingency + ContingentSize + ProbeColour + ProbeShape $+(1 \mid$ ProbeShape $)+(1 \mid$ ProbeColour $)+(1 \mid$ Subject $))$ in the lme4 syntax. It should be noted here that the effect of the mouse contingent condition was in the opposite direction than predicted with a $420 \mathrm{~ms}$ cost of untethered covert attention. This also contrasts with our previously found benefits from the change blindness paradigm. This advantage for the tethered condition starts as early as one or two seconds, and continues throughout a trial (see figure 3.b). The RT analyses also reveal that there is a speed/accuracy trade-off with relatively high error rates and fast RTs in the base, target-present condition as compared to both target absent and gaze contingent trials. We do not observe the same trade-off in the mouse aperture condition, since the accuracy scores are near ceiling for all mouse aperture conditions. 


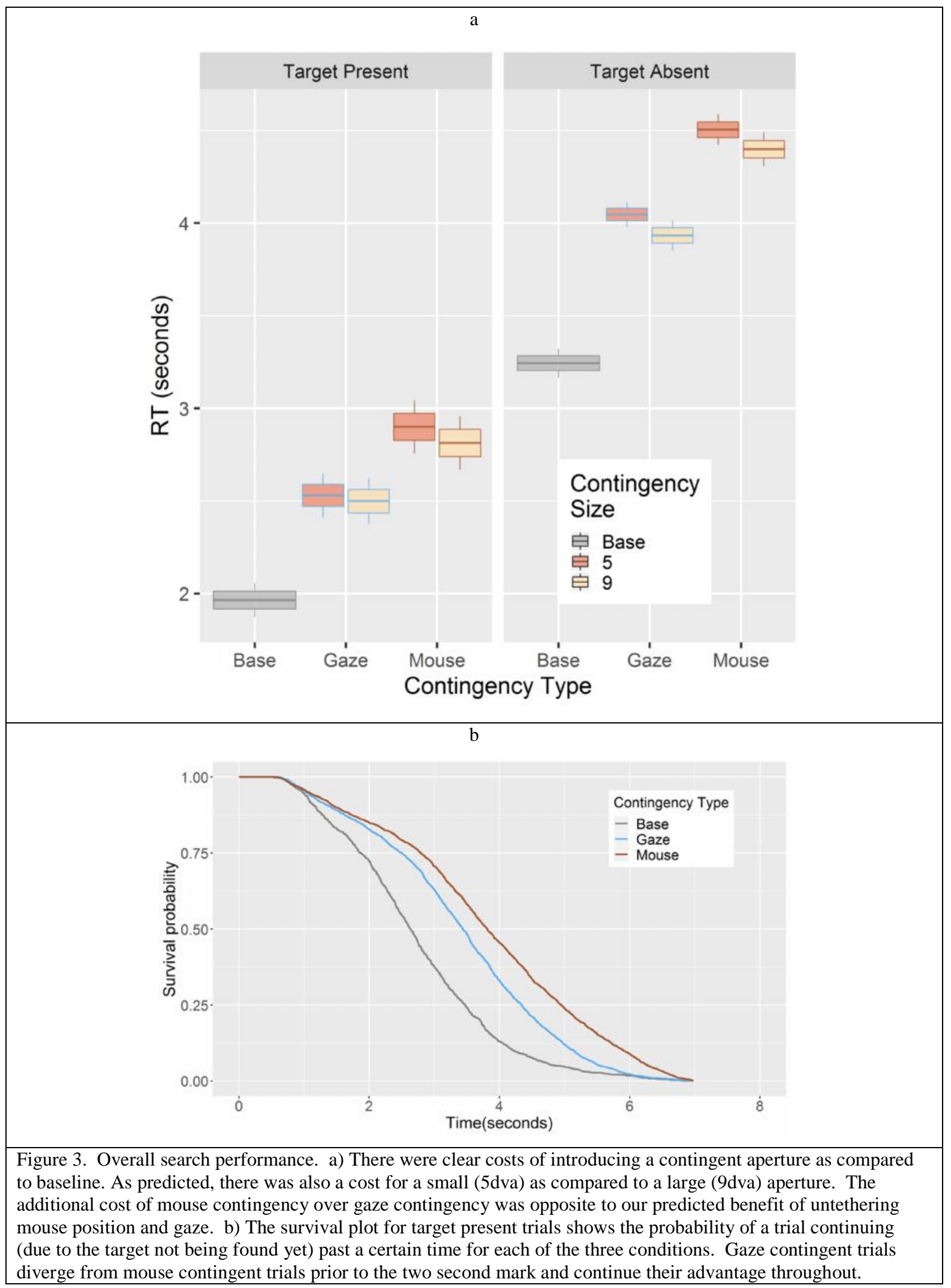

Evidence of untethered covert attention 
To determine if participants made any attempt to untether covert attention from the locus of gaze, we examined a number of mouse and gaze patterns. All analyses were run with linear mixed effects regression as above and included the same random effects. We should note that just because the mouse contingent condition allows the aperture centre to diverge from the centre of gaze, this does not guarantee that participants would adopt such a strategy. If participants adopted a strategy where they untethered the aperture to increase the use of covert attention, we would expect the gaze-mouse distance to increase. This would indicate that the mouse location is strategically separated from the centre of gaze as opposed to observers matching the focus of gaze and aperture as best they could. We suggest at least $2 \mathrm{dva}$ at minimum, which would place the centre of the aperture outside the range of the fovea centralis. We also predicted that this distance would be greater for the 9-degree mouse aperture than with the 5-degree mouse aperture since a larger potential area for covert attention was available.

The average Euclidean distance between gaze and mouse was $3.2 \mathrm{dva}(\mathrm{SE}=0.08)$. Including the size of the aperture improved the fit of the model $\left(\chi^{2}(1)=150, \mathrm{p}<.001\right)$ and resulted in an increase of Euclidean distance of $0.28 \mathrm{dva}$ $(\mathrm{SE}=0.02)$ for the larger aperture. This result clearly indicates that observers attempted to use this option of untethered covert attention.

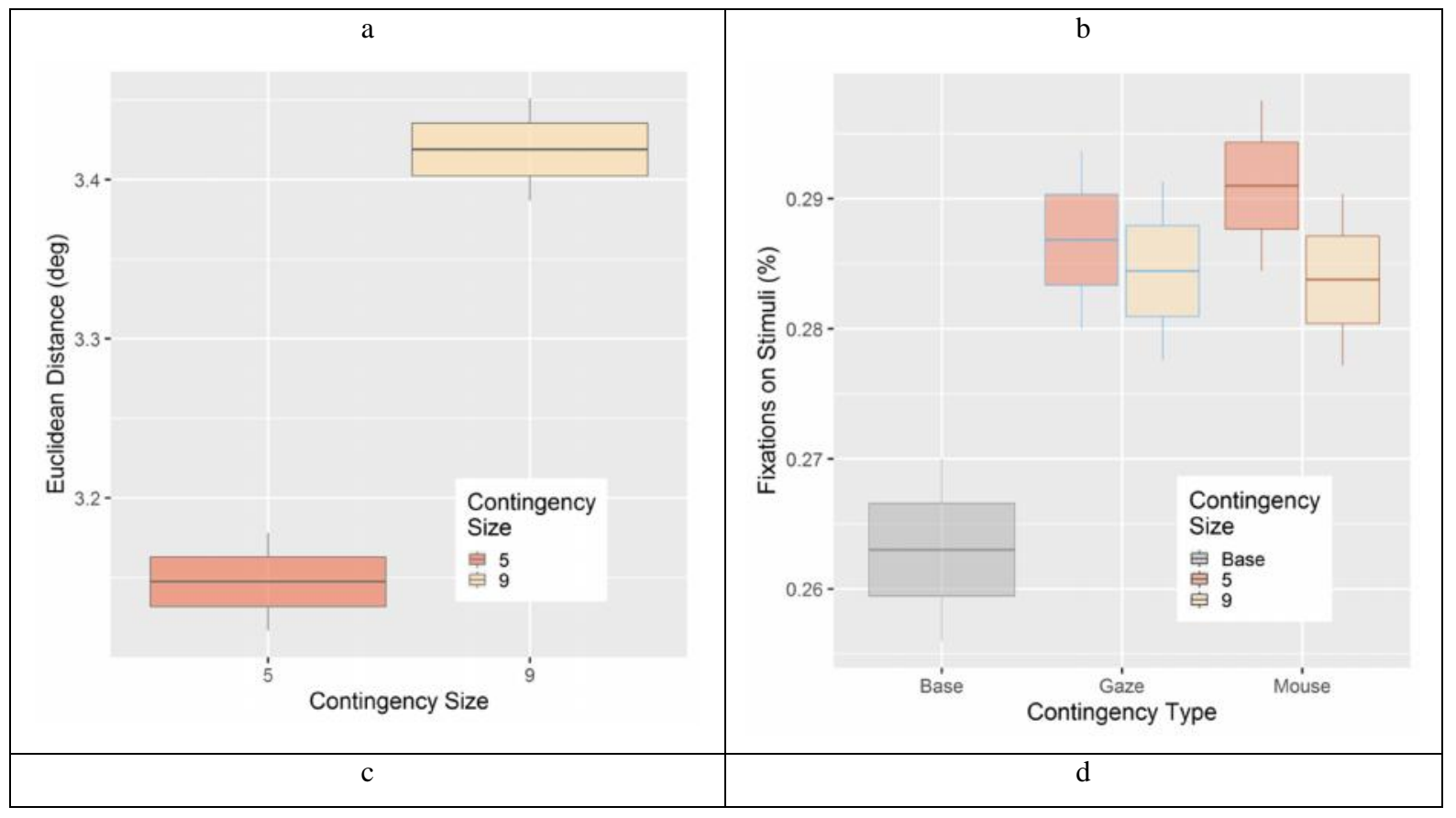




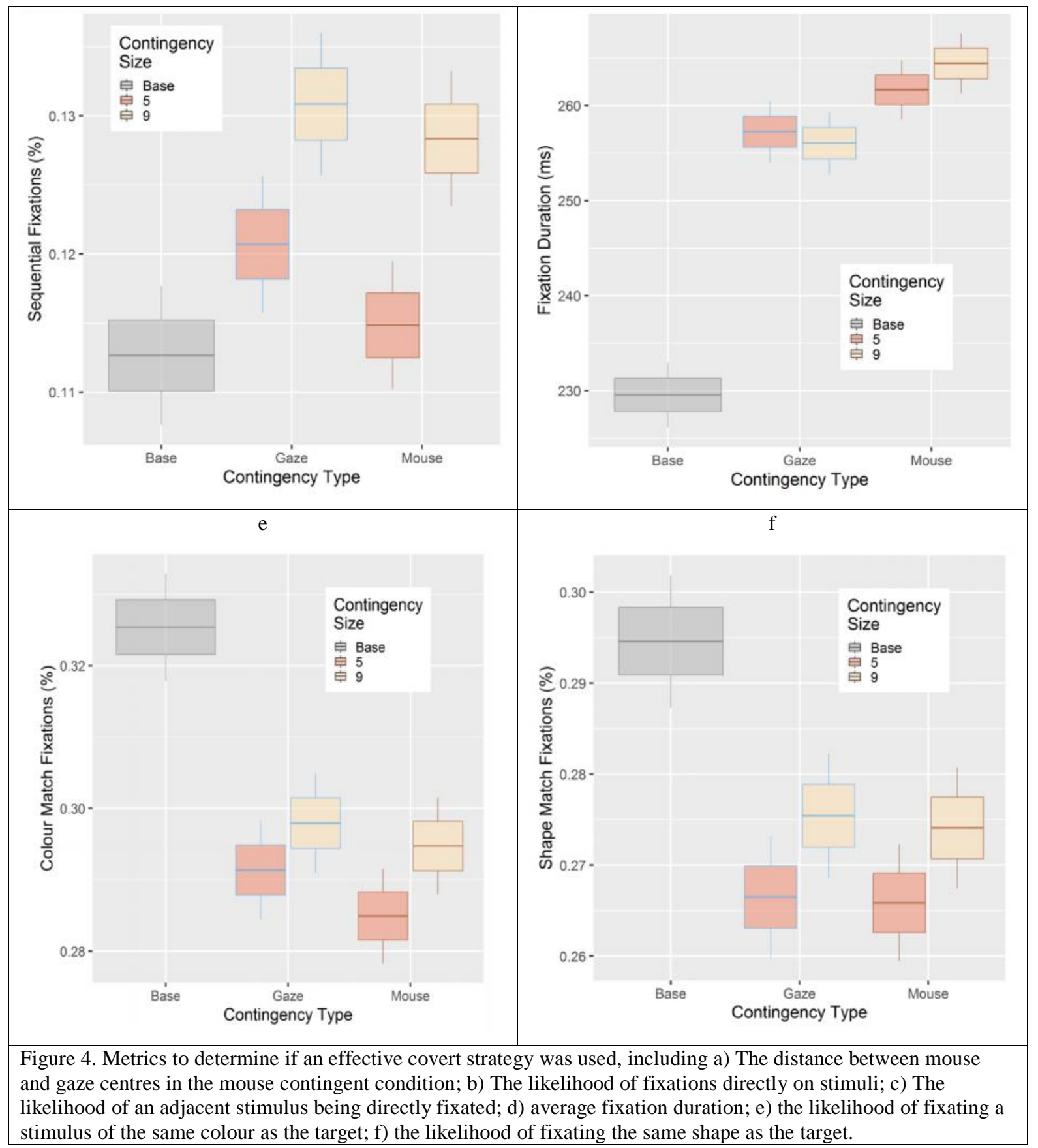

If the mouse contingent manipulation results in an increased use of covert attention, then fewer fixations directly on stimuli could reflect greater reliance on covert attention. Similarly, when observers do fixate stimuli, they may be less likely to do so when using a simple sequential scanning strategy similar to "reading" rows or columns. When we examined fixations that landed directly on stimuli, there was a main effect of aperture size, but it was only due to the baseline condition where participants were more likely to fixate on stimuli as compared to the two aperture conditions. There were neither interactions nor differences between aperture conditions and sizes. For the likelihood of fixating a directly adjacent stimulus (representing a more sequential scanning strategy), we found a main effect of aperture size with observers more likely to adopt this sequential strategy for the 9 dva apertures $(\chi 2(1)=20.6$, 
$\mathrm{p}<.001)$. The difference between gaze and mouse aperture type was not significant $\left(\chi^{2}(1)=3.5, \mathrm{p}=.062\right)$ but the trend was for fewer sequential fixations in the mouse condition.

Average fixation duration for a trial may reflect cognitive load during a task, but it may also reflect an increase in the use of covert attention reflected as the number of visual items being processed (Meghanathan et al., 2015). We observed a main effect of aperture type $\left(\chi^{2}(1)=12.4, \mathrm{p}<.001\right)$ with fixations on mouse contingent trials 4 ms slower $(\mathrm{SE}=2.2)$ than on gaze contingent trials. If this cost in fixation duration were caused by increased load, we would expect to see increased fixation durations for the smaller and more difficult 5-degree apertures but there was no effect of aperture size nor an interaction.

Our stimuli were selected so that they provide redundant information that could potentially be used to make the search more efficient. Namely, the colour and shape information of the target are non-essential information for the task, however, one or both could be used to limit the number of potential stimuli that need to be inspected on a given trial. For example, if the search target was a blue (colour), square (shape) with a tree inside (symbol) then participants could deploy covert attention to preselect only blue and/or square items for further selection, performing so-called subset search based only on these features (Egeth, Virzi \& Garbart, 1984; Friedman-Hill \& Wolfe, 1995). As such, we suggest that increased fixations on items that match the colour or shape of the target represent a successful attempt to deploy covert attention. In particular, if participants used covert attention to dismiss distractors based on colour or shape, they could thus focus overt attention directly on the remainder for a more efficient subset search. For the conditions with apertures, we observed main effects of aperture size on the likelihood of fixating the same shape $(\chi 2(1)=5.9, \mathrm{p}=.015)$ and the same colour $(\chi 2(1)=5.8, \mathrm{p}=.016)$. There was no effect of aperture type on shape $\left(\chi^{2}(1)=x, p=.83\right)$ while there was a non-significant trend for colour $\left(\chi^{2}(1)=3.1, p=.08\right)$. Further, the (nonsignificant) direction for both colour and shape was for fewer matching fixations in the mouse contingent condition, suggesting that if observers attempted to use covert attention to preselect subsequent stimuli, they were less successful at this.

\section{Discussion}

We used a new paradigm that enables the untethering of covert and overt attention to investigate visual search performance. We asked two main questions: 1) Do observers deploy covert attention separately when given this possibility during visual search, and 2) if covert attention is untethered, does this improve their performance? Five experimental conditions in a visual search task were tested using a within-subject design, where the area of an aperture and the method of aperture control were manipulated. As expected, the area of covert attention available influenced performance, with the full view baseline condition exceeding all aperture conditions, and performance with large apertures $(9 \mathrm{dva})$ better than for small ones $(5 \mathrm{dva})$. These results suggest that our contingent manipulation was successful in limiting the scope of covert attention, and that limiting covert attention negatively affected search performance. We also observed evidence for changes in search strategy with the mouse contingent aperture as compared to the gaze contingent one. Participants tended to maintain the aperture focal points more than 3 degrees away from the focus of gaze, and this separation increased with the larger apertures. Participants also had longer fixations in the mouse contingent condition. Taken together, the evidence suggests that participants attempted to use the mouse aperture untethered from the focus of gaze. Contrary to our hypothesis, however, separate control of the aperture by using the mouse resulted in worse search performance as measured by average response times during the search and fewer saccades to distractors with matching features. Survival analyses suggested that this 
advantage occurred early in the first second (figure 3) of the search and was maintained throughout the search duration.

Covert attention improves search performance for both feature and conjunction search (Carrasco \& Yeshuran, 1998) by increasing the rate by which information is processed at the attended location (Carrasco \& McElree, 2001). Covert attentional selection during serial conjunction search is likely directed by the Frontal Eye Fields (Buschman \& Miller, 2009) with possible additional contributions from other networks, including the supplementary eye fields (Corbetta \& Shullman, 1998) and parietal areas (Donner, et al. 2000). Although the premotor theory of attention suggests that covert attention is tied to the same network as for generating eye movements, activity within the FEF for the two has been shown to be independent (Thompson, Biscoe and Sato, 2005). If the two networks can operate independently, then there is potentially a benefit to be gained if the locus of covert attention can be separated from the locus of gaze. In fact, in a previous study with the change blindness paradigm we found such improved performance when participants were able to control a visible aperture separately from the centre of gaze (Chetverikov et al, 2018).

Although some tasks may benefit from an untethered spotlight of covert attention, our current visual search results align more closely with the Active Vision hypothesis (Findlay \& Gilchrist, 2001). They suggested that covert attention has benefits for tasks like search and reading, not through gaze independent selection of scene items, but primarily through the preview of subsequent fixation locations. For example, attention precedes a saccade to a spatial location (Hoffman and Subramaniam, 1995) and participants report the time of saccade arrival up to $80 \mathrm{~ms}$ prior to their actual arrival when saccading to a spinning clock (Hunt \& Cavanagh, 2009). Covert attention towards peripheral localization is also diminished during a saccade, suggesting a common, limited attentional resource (MacInnes \& Hunt, 2014). Remapping of a neurons receptive field just prior to a saccade (in lateral intraparietal cortex LIP, Colby, \& Goldberg, 1992; and in FEF, Sommer \& Wurtz, 2002) has been suggested as a neural mechanism to explain these behavioural results and has also been suggested as the primary mechanism to maintain visual stability across eye movements (Wurtz, 2008; Golumb, 2019).

Although we found no evidence that untethered covert attention led to a visual search benefit this presents a puzzle in light of our previous finding on performance on a change detection task where untethered attention led to improved performance (Chetverikov, 2018). We can speculate that it is detrimental to split attention between two loci in this visual search task and that the attentional demands of the change blindness and visual search tasks differ. Attentional capacity is limited and attempts at untethering may lead to costs in this case, one that did not surface in the change detection task. While there were a few differences between the studies, the most obvious was perhaps that during the change detection task the screen flickered with blank screens interleaved with the stimuli. This means that there were regular onsets throughout the task that could attract covert attention in a way similar to more controlled cuing studies. Another key difference was that the change blindness task involved a dynamic aperture that would shrink over time while not in motion. This may have had an impact on participant strategy, encouraging more frequent aperture movements to reset the aperture size. For example, it is possible that an increased frequency of saccades to maintain aperture size loads a common attentional resource needed for covert, peripheral updating via remapping (MacInnes \& Hunt, 2014).

Although our results suggest that observers tried to use the mouse contingent aperture condition to search independently of the locus of gaze, this resulted in fewer saccades to distractors that matched the target on one of the two additional features (shape and colour). The idea that attention might be allocated differently depending on the 
task is not new, for example Inhibition of return is observed in search tasks but not scene memorization or inspection ( Dodd, Van der Stigchel \&, Hollingworth, 2009) and eye movement patterns differ based on instruction (Yarbus, 1965; DeAngelus \& Pelz, 2009; Henderson et al, 2013; MacInnes et al, 2018). Given the evidence here that participants actively tried to use the untethered aperture, we suggest that the difference in results for search and change blindness was due to differences in the task. Another potential reason why this differs between visual search and change blindness might be that the two reflect different levels of perceptual processing and place differing demands upon visual attention. Overall, we can conclude that the mouse contingent task was sensitive to changes in aperture area suggesting that it carries promise for assessing covert attention. For untethering covert and overt attention, the next step will involve figuring out what explains the discrepancy.

\section{References}

1. Hoffman, J. E., \& Subramaniam, B. (1995). The role of visual attention in saccadic eye movements. Perception \& psychophysics, 57(6), 787-795.

2. Kowler, E., Anderson, E., Dosher B. \& Blaser, E. The role of attention in the programming of saccades. Vision Res. 35, 1897-1916 (1995).

3. Kustov, A. A. \& Robinson, D. L. Shared neural control of attentional shifts and eye movements. Nature 384, 74-77 (1996).

4. Desimone, R., \& Duncan, J. (1995). Neural mechanisms of selective visual attention. Annual review of neuroscience, 18(1), 193-222.

5. Driver, J. (2001). A selective review of selective attention research from the past century. British Journal of Psychology, 92(1), 53-78.

6. Egeth, H. (1977). Attention and preattention. In Psychology of Learning and Motivation (Vol. 11, pp. 277320). Academic Press.

7. Most, S. B., Scholl, B. J., Clifford, E. R., \& Simons, D. J. (2005). What you see is what you set: sustained inattentional blindness and the capture of awareness. Psychological review, 112(1), 217.

8. Kristjánsson, A. (2006). Simultaneous priming along multiple feature dimensions in a visual search task. Vision research, 46(16), 2554-2570.

9. Egeth, H. E., \& Yantis, S. (1997). Visual attention: Control, representation, and time course. Annual review of psychology, 48(1), 269-297.

10. Theeuwes, J. (2010). Top-down and bottom-up control of visual selection. Acta psychologica, 135(2), 77 99.

11. Posner, M. I. (1980). Orienting of attention. Quarterly journal of experimental psychology, 32(1), 3-25.

12. Deubel, H., \& Schneider, W. X. (1996). Saccade target selection and object recognition: Evidence for a common attentional mechanism. Vision research, 36(12), 1827-1837.

13. MacInnes, W.J. \& Hunt, A.R. Exp Brain Res (2014) 232: 3737. https://doi.org/10.1007/s00221-014-4062-2

14. Golomb, J. D. (2019). The what and when of remapping across saccades: a dual-spotlight theory of attentional updating and its implications for feature perception. Current Opinion in Psychology.

15. Klein, R. (1980). Does oculomotor readiness mediate cognitive control of visual attention?. Attention and performance, 8, 259-276.

16. Sheliga, B. M., Riggio, L., \& Rizzolatti, G. (1994). Orienting of attention and eye movements. Experimental Brain Research, 98(3), 507-522. 
17. Kristjansson, A. (2011). The intriguing interactive relationship between visual attention and saccadic eye movements. The Oxford handbook of eye movements, 455-470.

18. Klein, R. M., \& Pontefract, A. (1994). 13 Does Oculomotor Readiness Mediate Cognitive Control of Visual Attention? Revisited!. Attention and performance XV: Conscious and nonconscious information processing, 333 .

19. Hunt, A. R., \& Kingstone, A. (2003). Inhibition of return: Dissociating attentional and oculomotor components. Journal of Experimental Psychology: Human Perception and Performance, 29(5), 1068.

20. Sato, T. R., \& Schall, J. D. (2003). Effects of stimulus-response compatibility on neural selection in frontal eye field. Neuron, 38(4), 637-648.

21. Wollenberg, L., Deubel, H., \& Szinte, M. (2018). Visual attention is not deployed at the endpoint of averaging saccades. PLoS biology, 16(6), e2006548.

22. Smith, D. T., \& Schenk, T. (2012). The premotor theory of attention: time to move on?. Neuropsychologia, 50(6), 1104-1114.

23. Beauchamp, M. S., Petit, L., Ellmore, T. M., Ingeholm, J., \& Haxby, J. V. (2001). A parametric fMRI study of overt and covert shifts of visuospatial attention. Neuroimage, 14(2), 310-321.

24. Corbetta, M., Akbudak, E., Conturo, T. E., Snyder, A. Z., Ollinger, J. M., Drury, H. A., ... \& Shulman, G. L. (1998). A common network of functional areas for attention and eye movements. Neuron, 21(4), 761773.

25. De Haan, B., Morgan, P. S., \& Rorden, C. (2008). Covert orienting of attention and overt eye movements activate identical brain regions. Brain research, 1204, 102-111.

26. Armstrong, K. M., Fitzgerald, J. K., \& Moore, T. (2006). Changes in visual receptive fields with microstimulation of frontal cortex. Neuron, 50(5), 791-798.

27. Beckers, G., Canavan, A. G. M., Zangemeister, W. H., \& Homberg, V. (1992). Transcranial magnetic stimulation of human frontal and parietal cortex impairs programming of periodic saccades. Neuroophthalmology, 12(5), 289-295.

28. Grosbras, M. H., \& Paus, T. (2002). Transcranial magnetic stimulation of the human frontal eye field: effects on visual perception and attention. Journal of cognitive neuroscience, 14(7), 1109-1120.

29. Muggleton, N. G., Juan, C. H., Cowey, A., \& Walsh, V. (2003). Human frontal eye fields and visual search. Journal of neurophysiology, 89(6), 3340-3343.

30. Thompson, K. G., Biscoe, K. L., \& Sato, T. R. (2005). Neuronal basis of covert spatial attention in the frontal eye field. Journal of Neuroscience, 25(41), 9479-9487.

31. Rafal, R. D., Calabresi, P. A., Brennan, C. W., \& Sciolto, T. K. (1989). Saccade preparation inhibits reorienting to recently attended locations. Journal of Experimental Psychology: Human Perception and Performance, 15(4), 673.

32. Chica, A. B., Klein, R. M., Rafal, R. D., \& Hopfinger, J. B. (2010). Endogenous saccade preparation does not produce inhibition of return: failure to replicate Rafal, Calabresi, Brennan, \& Sciolto (1989). Journal of Experimental Psychology: Human Perception and Performance, 36(5), 1193.

33. Carrasco, M., \& McElree, B. (2001). Covert attention accelerates the rate of visual information processing. Proceedings of the National Academy of Sciences, 98(9), 5363-5367.

34. Chetverikov, A., Kuvaldina, M., MacInnes, W. J., Jóhannesson, Ó. I., \& Kristjánsson, Á. (2018). Implicit processing during change blindness revealed with mouse-contingent and gaze-contingent displays. Attention, Perception, \& Psychophysics, 80(4), 844-859. 
35. Duncan, J., \& Humphreys, G. W. (1989). Visual search and stimulus similarity. Psychological review, 96(3), 433.

36. Engbert, R., \& Kliegl, R. (2003). Microsaccades uncover the orientation of covert attention. Vision research, 43(9), 1035-1045.

37. Foulsham, T., \& Underwood, G. (2011). If visual saliency predicts search, then why? Evidence from normal and gaze-contingent search tasks in natural scenes. Cognitive Computation, 3(1), 48-63.

38. Geisler, W. S., Perry, J. S., \& Najemnik, J. (2006). Visual search: The role of peripheral information measured using gaze-contingent displays. Journal of Vision, 6(9), 1-1.

39. Kristjánsson, A. (2015). Reconsidering visual search. i-Perception, 6(6), 2041669515614670.

40. Nakayama, K., \& Martini, P. (2011). Situating visual search. Vision research, 51(13), 1526-1537.

41. Neisser, U. (1964). Visual search. Scientific American.

42. Corbetta, M., \& Shulman, G. L. (2002). Control of goal-directed and stimulus-driven attention in the brain. Nature reviews neuroscience, 3(3), 201.

43. Kristjánsson, Á., Sigurjónsdóttir, Ó., \& Driver, J. (2010). Fortune and reversals of fortune in visual search: Reward contingencies for pop-out targets affect search efficiency and target repetition effects. Attention, Perception, \& Psychophysics, 72(5), 1229-1236.

44. Kristjánsson, Á., \& Nakayama, K. (2003). A primitive memory system for the deployment of transient attention. Perception \& Psychophysics, 65(5), 711-724.

45. Friedman-Hill, S., \& Wolfe, J. M. (1995). Second-order parallel processing: Visual search for the odd item in a subset. Journal of Experimental Psychology: Human Perception and Performance, 21(3), 531.

46. Chelazzi, L., Perlato, A., Santandrea, E., \& Della Libera, C. (2013). Rewards teach visual selective attention. Vision research, 85, 58-72.

47. Awh, E., Belopolsky, A. V., \& Theeuwes, J. (2012). Top-down versus bottom-up attentional control: A failed theoretical dichotomy. Trends in cognitive sciences, 16(8), 437-443.

48. Kristjánsson, A., Wang, D., \& Nakayama, K. (2002). The role of priming in conjunctive visual search. Cognition, 85(1), 37-52.

49. Wolfe, J. M., \& Horowitz, T. S. (2017). Five factors that guide attention in visual search. Nature Human Behaviour, 1(3), 0058.

50. Bates, D., Kliegl, R., Vasishth, S., \& Baayen, H. (2015). Parsimonious mixed models. arXiv preprint arXiv:1506.04967.

51. Egeth, H. E., Virzi, R. A., \& Garbart, H. (1984). Searching for conjunctively defined targets. Journal of Experimental Psychology: Human Perception and Performance, 10(1), 32.

52. Yeshurun, Y., \& Carrasco, M. (1998). Attention improves or impairs visual performance by enhancing spatial resolution. Nature, 396(6706), 72.

53. Carrasco, M., \& McElree, B. (2001). Covert attention speeds the accrual of visual information. Proceedings of the National Academy of Sciences, USA, 98, 5363-5367.

54. Buschman, T. J., \& Miller, E. K. (2009). Serial, covert shifts of attention during visual search are reflected by the frontal eye fields and correlated with population oscillations. Neuron, 63(3), 386-396.

55. Donner, T., Kettermann, A., Diesch, E., Ostendorf, F., Villringer, A., \& Brandt, S. A. (2000). Involvement of the human frontal eye field and multiple parietal areas in covert visual selection during conjunction search. European Journal of Neuroscience, 12(9), 3407-3414. 
56. Findlay, J. M., \& Gilchrist, I. D. (2001). Visual attention: The active vision perspective. In Vision and attention (pp. 83-103). Springer, New York, NY.

57. Hoffman, J. E., \& Subramaniam, B. (1995). The role of visual attention in saccadic eye movements. Perception \& psychophysics, 57(6), 787-795.

58. Hunt, A. R., \& Cavanagh, P. (2009). Looking ahead: The perceived direction of gaze shifts before the eyes move. Journal of Vision, 9(9), 1-1.

59. Colby, C. L., \& Goldberg, M. E. (1992). The updating of the representation of visual space in parietal cortex by intended eye movements. Science, 255(5040), 90-92.

60. Sommer, M. A., \& Wurtz, R. H. (2002). A pathway in primate brain for internal monitoring of movements. Science, 296(5572), 1480-1482.

61. Von Helmholtz, H. (1867). Handbuch der physiologischen Optik (Vol. 9). Voss.

62. Wurtz, R. H. (2008). Neuronal mechanisms of visual stability. Vision research, 48(20), 2070-2089.

63. Dodd, M. D., Van der Stigchel, S., \& Hollingworth, A. (2009). Novelty is not always the best policy: Inhibition of return and facilitation of return as a function of visual task. Psychological Science, 20(3), $333-$ 339.

64. Yarbus, A. L. (1965). Role of eye movements in the visual process.

65. DeAngelus, M., \& Pelz, J. B. (2009). Top-down control of eye movements: Yarbus revisited. Visual Cognition, 17(6-7), 790-811.

66. MacInnes, W. J., Hunt, A. R., Clarke, A. D., \& Dodd, M. D. (2018). A generative model of cognitive state from task and eye movements. Cognitive computation, 10(5), 703-717.

67. Meghanathan, R. N., van Leeuwen, C., \& Nikolaev, A. R. (2015). Fixation duration surpasses pupil size as a measure of memory load in free viewing. Frontiers in human neuroscience, 8, 1063.

68. Henderson, J. M., Shinkareva, S. V., Wang, J., Luke, S. G., \& Olejarczyk, J. (2013). Predicting cognitive state from eye movements. PloS one, 8(5), e64937.

\section{Appendix a}

Matlab code for aperture

appertureSize $\quad=$ floor(visArea* pixelsperdegree);

transLayer=2;

$[\mathrm{x}, \mathrm{y}]=$ =meshgrid(-appertureSize: appertureSize, - appertureSize:);

maskblob $=$ ones $(2 *$ appertureSize $+1,2 *$ appertureSize +1 , transLayer $) *$ grey;

\% Layer 2 (Transparency aka Alpha) is filled with gaussian transparency

$\%$ mask.

xsd= appertureSize $/ 2.2$;

ysd= appertureSize /2.2;

maskblob(:,:,transLayer $)=\operatorname{round}\left(255-\exp \left(-\left((\mathrm{x} / \mathrm{xsd}) .{ }^{\wedge} 2\right)-\left((\mathrm{y} / \mathrm{ysd}) .^{\wedge} 2\right)\right) * 255\right)$; 